\section{La universidad pública y su contribución a la formación de líderes guaraníes. El caso de los maestros bilingües de Salta}

Ana Victoria Casimiro Córdoba

Instituto de Investigaciones en

Ciencias Sociales y Humanidades.

Consejo Nacional de Investigaciones

Científicas y Técnicas. Universidad

Nacional de Salta, Argentina.

anita.desafio@gmail.com
A 100 años de la Reforma Universitaria de 1918

Intervenciones

RECEPCIÓN: 29/06/17

ACEPTACIÓN FINAL: 24/09/17

\section{Resumen}

El presente trabajo indaga la injerencia de la universidad pública y los proyectos de extensión en la formación de líderes comunitarios y gestores de la lengua nativa a partir de tres casos registrados en las comunidades originarias de la región del umbral al chaco salteño (Argentina): Yacuy, Tuyunti y Misión Cherenta. En primer lugar, hacemos referencia a los pueblos guaraníes de la región señalando algunos aspectos etnohistóricos y los procesos recientes de reconfiguración étnica; en segundo lugar, reseñamos el desarrollo de la Educación Intercultural Bilingüe (EIB) y los principales actores involucrados; y en tercer lugar, presentamos las experiencias de tres líderes originarios y su vinculación con la universidad pública y los proyectos de extensión. Finalmente, realizamos aportes al debate sobre la extensión universitaria y los liderazgos indígenas desde una propuesta orientada a la disminución de las brechas de desigualdad entre los diferentes actores, de modo de fortalecer los esfuerzos colectivos y el trabajo en red.

\section{Palabras clave}

- Universidad

- Extensión

- Líderes guaraníes

- Educación intercultural bilingüe

\section{Resumo}

Este artigo investiga a interferência dos projectos universitários e extensão públicas na formação de líderes comunitários e gerentes da língua nativa, a partir de três casos em comunidades indígenas da região limiar do Chaco salteño (Argentina): Yacuy, Tuyunti e Missão Cherenta.

Primeiro, nós nos referimos aos povos Guaraní do etno-histórica região apontando alguns aspectos e processos de reconfiguração étnicos recentes; em segundo lugar, rever o desenvolvimento do EIB e as principais partes interessadas; e em terceiro lugar, apresentamos as experiências de três líderes indigenas e as suas ligações com os projetos universitários e de extensão públicos. Finalmente, fez contribuições para o debate de extensão universitária e líderes indígenas a partir de uma proposta destinada a reduzir as desigualdades entre os diferentes atores, fortalecendo os esforços coletivos.

Palavras-chave

- Universidade

- Extensão

- Líderes guaraníes

- Educação bilíngue intercultural.
Para citación de este artículo

Casimiro Córdoba, A. V. (2017). La universidad pública y su contribución a la formación de líderes guaraníes. El caso de los maestros bilingües de Salta. Revista +E versión en línea, 7(7), 254-269. Santa Fe, Argentina: Ediciones UNL. 


\section{Introducción}

El presente trabajo indaga la injerencia de la universidad pública y los proyectos de extensión en la formación de líderes comunitarios y gestores de la lengua nativa a partir de la descripción y análisis de algunos aspectos de la implementación de la Educación Intercultural Bilingüe (EIB) en tres comunidades de origen guaraní del departamento San Martín de la región norte de la provincia de Salta. En primer lugar, se hace referencia a los pueblos guaraníes de la región señalando algunos aspectos etnohistóricos y los procesos recientes de reconfiguración étnica que influyen en las prácticas y discursos vinculados a la EIB. Luego se reseña el desarrollo de la (EIB) en Argentina y se focaliza en la provincia de Salta, se señalan los principales actores involucrados (comunidades, organizaciones intermedias, las ONG, universidades y maestros bilingües), se describen algunos entramados sociales y se identifican las problemáticas generales. En tercer lugar, se presentan las experiencias de tres líderes originarios, se describen algunos hitos en sus trayectorias personales y educativas que nos permiten indagar su vinculación con la universidad pública y los proyectos de extensión. A modo de cierre, realizamos aportes al debate sobre la extensión universitaria y los liderazgos indígenas desde una propuesta orientada a la disminución de las brechas de desigualdad entre los diferentes actores, de modo de fortalecer los esfuerzos colectivos y el trabajo en red.

\section{Espacio, etnohistoria e identidad}

Desde tiempos prehispánicos, el pueblo guaraní se ha expandido desde la selva amazónica hasta llegar al piedemonte andino, donde entabló relaciones políticas y de parentesco con las poblaciones locales, dando lugar al surgimiento de nuevos grupos "mestizos". A mediados del siglo XVI, los chiriguanos ${ }^{1}$ ya se habían conformado como un grupo étnico diferenciado que habitaba el piedemonte andino y mantenían relaciones conflictivas con el Imperio Incaico al que resistieron. La ocupación guaraní del espacio es, como vemos, previa a la formación de los Estados nacionales y la conformación de una frontera argentino-boliviana. En la Figura 1 se observa con claridad que esta región constituye actualmente una de las tres grandes áreas del territorio guaraní continental.

Figura 1: Guaraní Retã (EMGC, 2016)

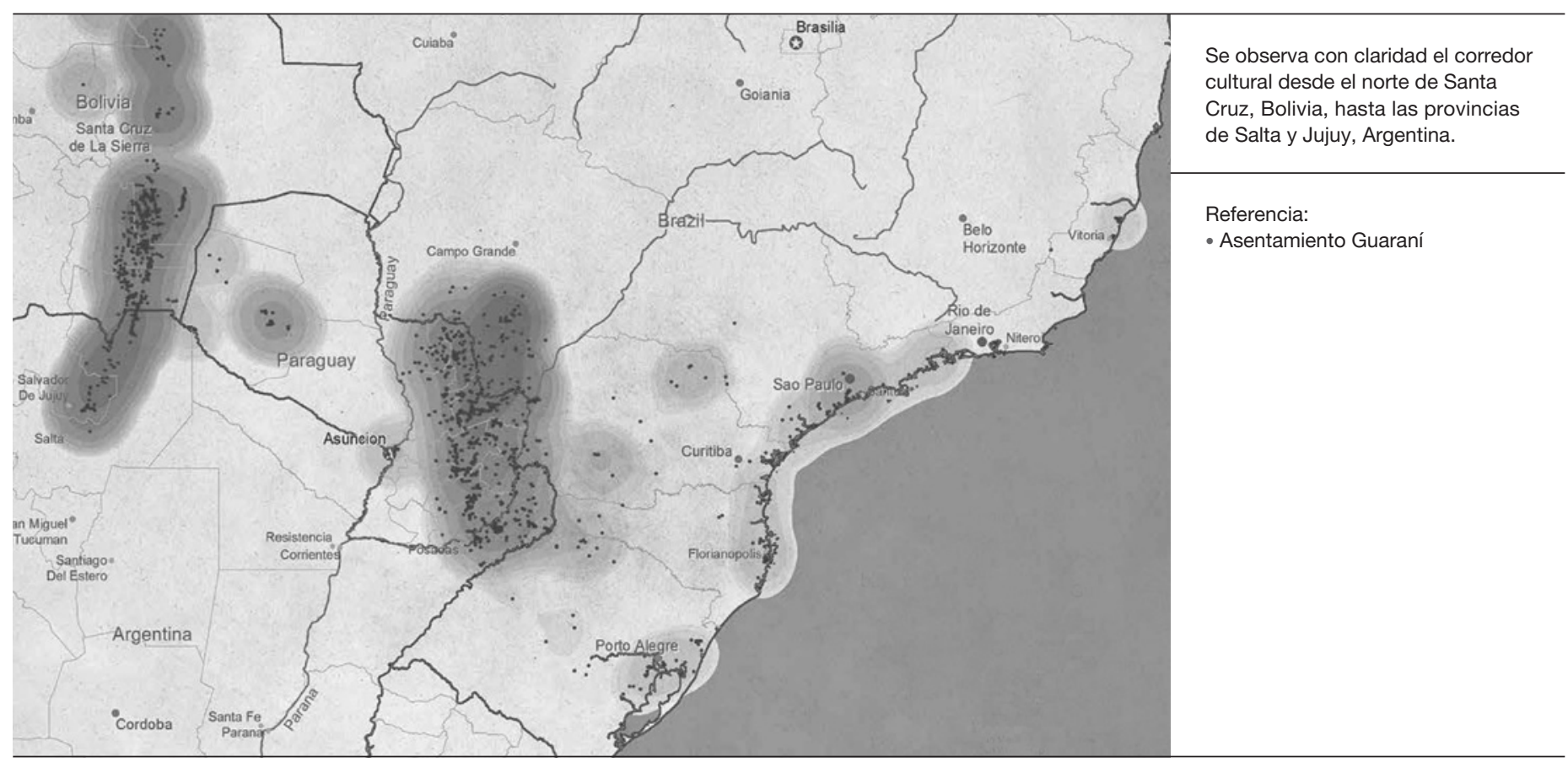

1) Más allá de la discusión acerca del origen del término, este parece haber designado a un grupo mestizo entre tupi guaraníes y pueblos chaquenses (guanas y chané) que conservaba la lengua guaraní pero con nuevas pautas culturales. 
En Argentina, los guaraníes chiriguanos se instalaron en el piedemonte o umbral al Chaco, en las provincias de Salta y Jujuy muy vinculadas con las poblaciones bolivianas, conformando un corredor cultural que conecta a su vez las tierras altas y bajas. Dentro del territorio argentino se pueden identificar tres procesos sociohistóricos que configuran la distribución actual de los pueblos guaraníes. En primer lugar, la migración laboral hacia los ingenios, que contrajo la instalación de una gran cantidad de población en las márgenes de las ciudades a lo largo del siglo XIX. En segundo lugar, la instalación de misiones franciscanas desde fines del siglo $\mathrm{XIX}$ y hasta primera mitad del XX, ${ }^{2}$ las cuales trajeron aparejada una transformación profunda de una serie de pautas socioculturales, como la sedentarización, la cristianización, la escolarización, la castellanización y la pérdida de la lengua nativa. En tercer lugar, la Guerra del Chaco entre Bolivia y Paraguay (1932-1935) produjo el desarraigo de muchísimas familias que, finalmente, terminaron refugiándose en Salta y Jujuy. ${ }^{3}$ Finalmente, luego del auge de los ingenios la mano de obra se ha volcado principalmente a la producción maderera y porotera. La circulación entre ambos países es constante, y se sostiene en redes de parentesco, la unidad lingüística y la conciencia común de una identidad compartida. En efecto, en el transitar entre Bolivia y Argentina los pueblos se reconocen como hermanos bajo la macrocategoría "guaraní", aunque conservan algunas diferencias. Con el paso del tiempo se dieron procesos de organización comunitaria, coexistiendo la generación de nuevas categorías étnicas con procesos de negación de la identidad originaria. ${ }^{4}$ Actualmente, en el lado argentino existen cuatro grupos de lengua guaraní: ava guaraní (llamados chiriguanos o guaraníes en Bolivia), tapiete, chané y tupi guaraní.

Para el caso del departamento San Martín, debemos remarcar la relación histórica establecida entre las etnias chané, isoseños y ava guaraní. Muchas de las familias que viven en comunidades a la orilla de la ruta nacional 34 , provienen de la zona del Isoso (Bolivia) antiguamente región chané (del tronco arawak) que atravesó un proceso de guaranízación lingüística y cultural. Cuando los guaraníes isoseños migraron al departamento San Martín (Salta, Argentina) no emplearon la identidad étnica chané ni se adscribieron al término ava guaraní, sino que recurrieron a un neologismo, tupi guaraní, que ya ha sido reconocido por el Estado nacional. Los tupi guaraní y los chané de Argentina, si bien hablan la lengua guaraní, mantienen fronteras étnicas con los ava guaraní, por lo que se trata de tres grupos cultural y lingüísticamente emparentados pero étnicamente diferenciados.

\section{Educación Intercultural Bilingüe, el caso de tres comunidades guaraníes}

La EIB se desarrolló en Argentina a partir de experiencias aisladas tales como las desarrolladas en las provincias de Chaco (año), Salta (año) y Formosa (año) (Serrudo, 2011), luego inició un proceso de institucionalización que tiene su punto más álgido en la Ley de Educación de 2006 que la instituye como modalidad educativa. ${ }^{5}$ En líneas generales, la aplicación de la EIB en el territorio nacional presenta varias problemáticas estructurales tales como a) la disparidad entre lo rural y lo urbano, b) la discontinuidad entre niveles educativos primario/secundario, c) el lugar secundario de la lengua indígena frente a la castellana dentro del aula, d) las tensiones entre maestros bilingües y el equipo docente no indígena, e) la poca participación de las comunidades en la toma de decisiones, f) la falta de presupuesto y de material pedagógico (Casimiro Córdoba y Flores, 2016).

Según el Relevamiento Anual de la Red Federal de Información Educativa, las áreas del sur andino, noroeste argentino (NOA) y noreste argentino (NEA) son las que más establecimientos concentran.

La provincia de Salta, perteneciente a la región del NOA, fue pionera en el desarrollo de la EIB $^{6}$ y en la incorporación de maestros bilingües en las escuelas. Las escuelas públicas con modalidad EIB se han concentrado en la región norte (departamento de Iruya, Santa Victoria, Orán, San Martín y Rivadavia), la cual posee la mayor población indígena y la mayor diversidad lingüística de la provincia. Las tres comunidades que aquí analizaremos pertenecen a la región norte de la provincia de Salta y se caracterizan por su rol protagónico en el desarrollo de la EIB, tanto por los intelectuales
2) En 1923 se creó la Custodia Provincial de Misioneros franciscanos de Salta que estableció misiones en la zona, algunas dieron lugar a comunidades actuales tales como: Río Caraparí (1933), La Loma (1942), Tuyunti (1944), San José de Yacuy (1952), Piquirenda (1964) y San Francisco de
Pichanal (1964).

3) Durante la Guerra del Chaco (1932-1935), los guaraníes ayudaron a sus hermanos guaraníes paraguayos, repitiendo la historia de sus luchas históricas contra los incas, se volvieron enemigos de la población boliviana que hablaba quechua y aimara. En este contexto muchos guaraníes de Bolivia huyeron a la Argentina. 4) De acuerdo a técnicos del Ministerio de Educación de la Provincia, etnógrafos y las comunidades ava guaraníes, la zona de Acambuco, limítrofe con Bolivia, está poblada por guaraníes aunque estos no se reconozcan como tales.
5) Algunos hitos legales en EIB son: en 1993, la Reforma Educativa que comenzó a dar nuevas pautas para la educación indígena; en 1997, el proyecto de Atención de las Necesidades Educativas de la Población Aborigen; en los años 2002 y 2003, la constitución de un equipo específico para la 
indígenas y la producción de materiales, como por la articulación que han establecido con las organizaciones intermedias indígenas y no indígenas, estatales y no estatales.

La Comunidad de Yacuy se ubica en un entorno rural dentro del municipio de Tartagal, la misma se autoconsidera tupi guaraní, sin embargo, es reconocida por las otras comunidades como guaraní isoseña. En efecto, esta comunidad mantiene relaciones de parentesco con comunidades del Isoso (Bolivia) y suelen intercambiar visitas, en una identificación geográfica que traspasa las fronteras (Hirsch, 2004). La variante guaraní que hablan es minoritaria en la zona pero posee una gran vitalidad en todos los grupos de edad. Yacuy cuenta con una Escuela y varios maestros bilingües que se desempeñan en el nivel primario. La comunidad ha sido destinataria y partícipe de una serie de proyectos de investigación y extensión universitaria como de la intervención de técnicos y las ONG, muchos de los cuales estuvieron vinculados a la EIB. Estas articulaciones han fortalecido los vínculos intercomunitarios marcando la emergencia de jóvenes líderes e intelectuales originarios de amplia trayectoria.

La Comunidad Tuyunti de la etnia Chané pertenece al Municipio de Aguaray y forma parte del ejido urbano. Cuenta con una escuela y un equipo de maestros bilingües desde fines de los ' 80 . La influencia de los franciscanos y Equipo Nacional de Pastoral Aborigen (ENDEPA) fue muy importante en la formación de los jóvenes líderes como educadores y difusores de su cultura. Si bien la lengua chané es muy vital, en los últimos años presenta un problema en la transmisión intergeneracional, pues los padres más jóvenes no la transmiten a sus hijos. Los alumnos ingresan a la escuela siendo bilingües en castellano y chané, aunque hay un fuerte avance de monolingüismo castellano en las nuevas generaciones. Tuyunti ha sido objeto y partícipe de muchas investigaciones y proyectos, a partir de los cuales se ha convertido en una de las comunidades más influyentes en temas educativos y sus intelectuales originarios son muy reconocidos.

Misión Cherenta por su parte es una comunidad periurbana de Tartagal, que se conformó como un desprendimiento de la comunidad urbana 9 de julio (Tartagal, Salta). La mayoría de su población se considera y es considerada por otros como ava guaraní, esta variante es la más extendida en la región. Al igual que otras comunidades rodeadas por barrios criollos, Cherenta presenta un proceso muy marcado de pérdida de la lengua puesto que solo es hablada por los adultos y mayores. Los niños ingresan a la escuela siendo monolingües en castellano aunque conociendo algunas palabras y frases en guaraní. Si en Yacuy se habla de una educación bilingüe orientada a alfabetizar en ambos idiomas, el caso de Cherenta implica el aprendizaje del guaraní como segunda lengua y el aprendizaje de su sistema de escritura con fines de comprensión básica orientado a la traducción. ${ }^{7}$ De hecho, en Cherenta los maestros bilingües hablan de un proceso de "mantenimiento" de la lengua, es decir, de darla a conocer en sus rudimentos "para que no se pierda", pero sin pretensiones de que sea incorporada en el habla cotidiana. ${ }^{8}$ A diferencia de Tuyunti y Yacuy, Misión Cherenta no ha sido el foco de las intervenciones de actores externos, sin embargo, cuenta con un equipo de maestros bilingües sumamente activo y en la actualidad atraviesa un proceso muy peculiar de formación y empoderamiento de los maestros a partir de la autogestión colectiva.

De Yacuy y Tuyunti surgieron los representantes guaraní y chané del Consejo Educativo Autónomo de los Pueblos Indígenas (CEAPI) y de Misión Cherenta el presidente de la recientemente creada Confederación de Docentes Guaraní (CDG). Estas organizaciones intermedias son claves para la implementación de la EIB.

\section{La EIB desde las organizaciones intermedias}

Cuando se produjo discusión de la Ley de Educación Nº 26.206 del año 2006, el Estado nacional argentino creó un Consejo Educativo Autónomo de Pueblos Indígenas (CEAPI) con función consultiva y de asesoramiento ante el Ministerio de Educación Nacional y el Consejo Federal de Educación. El CEAPI se define en sus constituciones como un espacio autónomo y autárquico para la toma de decisiones participativas y consensuadas en materia de educación, ${ }^{9}$ en el marco formal posee un rol consultivo, brindando consejo a los encargados oficiales de la aplicación
Población Intercultural; en el año 2004 el Programa Nacional de Educación Intercultural Bilingüe (PNEIB) y en 2006 la transformación de dicho programa en Modalidad Educativa. 6) En 1983-1984 la provincia ya contaba con un Consejo General de Educación de Salta que implemen- tó el Proyecto de Regionalización Educativa para el Área Aborigen (PREPARA) orientado hacia una Educación Bicultural. Luego, en 1995 se comenzó a implementar, un proyecto de educación bilingüe auspiciado por el Ministerio de Educación de la Provincia y ENDEPA (Equipo Nacional de Pastoral Aborigen). Finalmente en el año 2008 la Ley $N^{\circ} 7.546$, siguiendo la legislación nacional, estableció la EIB como modalidad del sistema educativo provincial.

7) Las diferencias entre estas comunidades son explicadas por Hirsch (2004) como consecuencia de las relaciones diferentes que los ava y los isoseños mantuvieron con la Iglesia, el Estado y el poder económico.

8) Entrevista a Gabriel, Misión Cherenta, abril de 2016.

9) Constitución del CEAPI disponible en pagina web oficial: http://www. ceapi.info/ 
de la EIB, quienes definen los marcos formales y los contenidos curriculares y, en última instancia, toman las decisiones.

EI CEAPI ha iniciado recientemente un proceso de ampliación, por una parte, incorporando nuevos representantes de provincias y pueblos que inicialmente no fueron convocados, y por otra, incluyendo una mayor proporción de mujeres (sector históricamente minoritario en las esferas de poder indígena).

Pese a estos procesos, luego de diez años de gestión, la entidad presenta tanto una crisis institucional vinculada a las dificultades logísticas y materiales como una crisis de representatividad asociada a los mecanismos de elección de los referentes nacionales del Consejo, y a procesos más generales de distanciamiento de los intelectuales orgánicos respecto de las problemáticas locales y los problemas de comunicación con sus principales interlocutores: los maestros bilingües y la comunidad. El proceso de provincialización del CEAPI, ya iniciado por Córdoba, Jujuy, Chubut y aún en marcha en Salta, ${ }^{10}$ se presenta pues como una necesidad interna de la entidad. La coexistencia de un CEAPI nacional con los CEAPI provinciales busca mejorar la llegada de la organización a las localidades fortaleciendo la comunicación entre los referentes y las comunidades.

En este contexto, un grupo de maestros bilingües guaraníes de los departamentos San Martín (Tartagal, Aguaray y Salvador Maza) y Orán (Pichanal) con demandas, intereses y expectativas comunes se reunieron para conformar la Confederación de Docentes Guaraníes en EIB de San Martín y Orán (CDG). Se trata de un proceso de organización colectiva y autogestionada que cuenta con el apoyo del Instituto Provincial Indígena de Salta (IPIS), la Asamblea del Pueblo Guaraní (APG-Sede Argentina) y con el acompañamiento de dos investigadoras de la Universidad Nacional de Salta. La emergencia de la CDG apunta a democratizar el acceso a la información, canalizar las demandas del sector y gestionar nuevas estrategias de trabajo en temas educativos. La función de la misma no es meramente consultiva, ya que entre las primeras actividades propuestas por la entidad se encuentran las capacitaciones dirigidas a los propios maestros, en áreas tales como gramática, medicina, literatura, música y gastronomía guaraní, como así capacitaciones hacia la comunidad educativa y el público general. Otra de las actividades propuestas es recopilar el material pedagógico (cartillas, libros, planes de trabajo, programas, contenidos audiovisuales, etc.) realizado de manera individual y grupal en diferentes y dispersas comunidades, para generar un fondo común y línea de base para la construcción de un currículo guaraní, la publicación de libros y cartillas para todas las escuelas de la región.

Mientras el CEAPI fue creado desde el Estado hacia las comunidades, la CDG fue construida en sentido inverso, justamente con el objeto de transformar los lugares de enunciación de los maestros bilingües y las condiciones de participación frente al Estado (provincial y nacional). Ambas se han apropiado de la normativa vigente que ampara los derechos constitucionales de los pueblos originarios a recibir una educación intercultural bilingüe. Más allá de las concepciones académicas y estatales de la EIB como paradigma o modelo educativo, estas organizaciones la consideran un verdadero proyecto político. Desde algunos sectores del CEAPI se ha planteado que la EIB puede ser el inicio de un camino hacia la Educación Autónoma de los pueblos originarios, señalando su lugar en la construcción de una nueva ciudadanía que contemple la diversidad cultural y las identidades múltiples, en definitiva, nuevas ciudanías interculturales (Machaca, 2011). La CDG, por su parte, ha comenzado a ejercer el derecho a la interculturalidad, concebido como una praxis de autogestión colectiva, se trata de construir la EIB a la medida del pueblo guaraní y desde los saberes culturales y las competencias educativas adquiridas por los propios maestros bilingües a lo largo de tres décadas.

La articulación de ambas organizaciones es una tarea que los maestros bilingües de la zona han comenzado a encarar, en un contexto nacional de retroceso en materia de derechos y políticas públicas. ${ }^{11}$ En este sentido, estas organizaciones se han constituido en voces defensoras del derecho constitucional a la EIB, postulado como un derecho eminentemente indígena. ${ }^{12}$

Las ONG y las universidades públicas. Contribuciones a la EIB Además del Estado provincial y nacional, dos actores externos a las comunidades destacan por su injerencia en el desarrollo educativo y cultural de la región: las ONG y las universidades públicas. Ambas fueron fundamentales en la activación y fortalecimiento de los tejidos sociales y la generación de líderes originarios.
10) El Ministerio de Educación de la Provincia, desde la Coordinación de la Modalidad EIB ha iniciado las gestiones necesarias para conformar un CEAPI.

11) Desde el gobierno nacional se orquestó una reestructuración del
Ministerio de Educación y una redefinición de la partida presupuestaria para educación, lo cual hizo clara la intención de suprimir la Modalidad de Educación Intercultural Bilingüe (EIB) y puso en riesgo los derechos constitucionales de los Pueblos Originarios.
12) Los sentidos sociales de la interculturalidad se encuentran en pugna entre los pueblos originarios y otros actores en el contexto nacional; la apropiación de los primeros se vincula a sus luchas históricas y a la experiencia boliviana. 


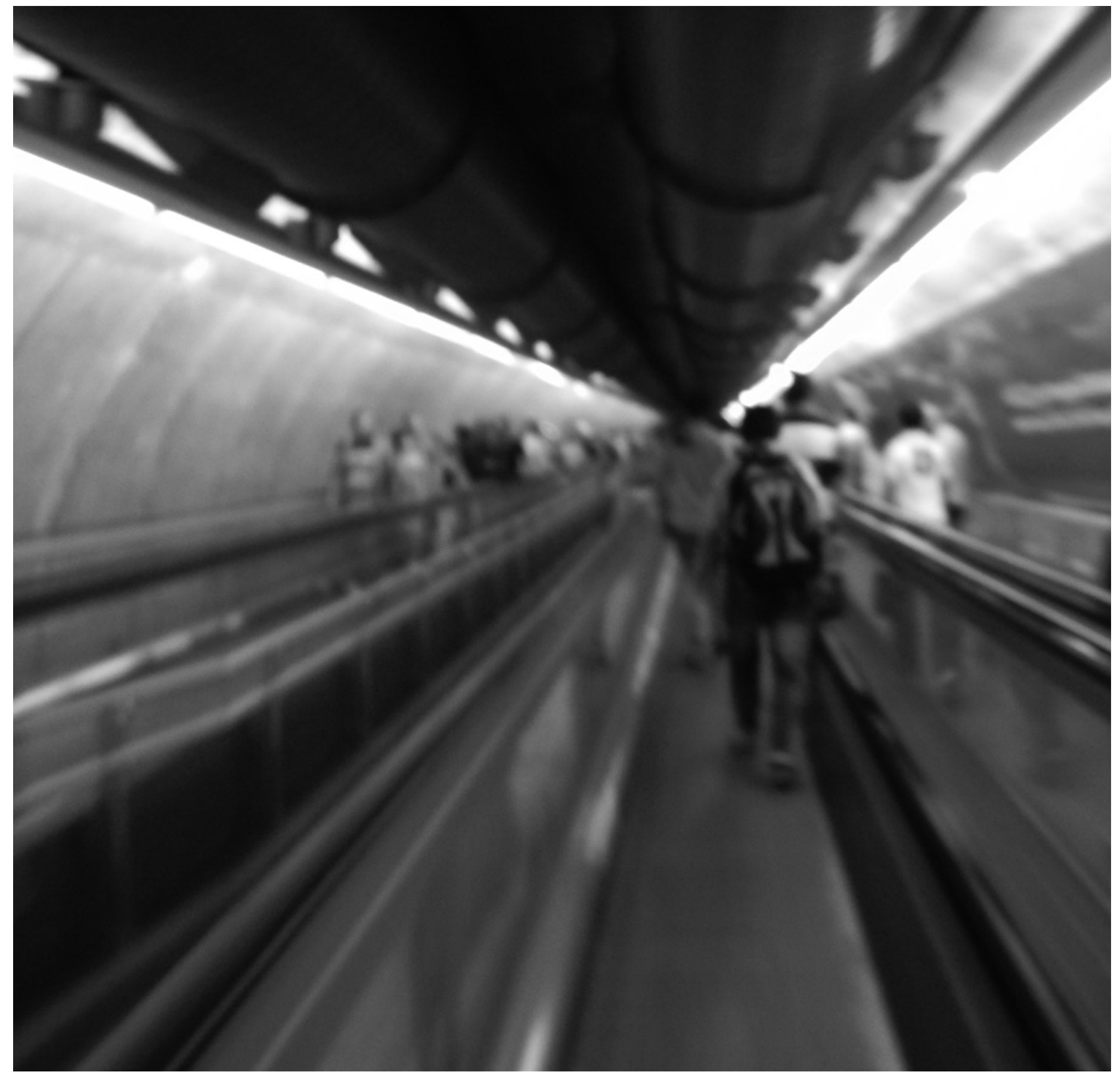

() Cecilia luco 
Son varias y variadas las ONG que trabajan en la región, ${ }^{13}$ las mismas entablaron relaciones duraderas con las comunidades. Castelnuovo Biraben (2010) ha señalado un primer momento en esta interacción caracterizado por una lógica de dar y recibir que asignaba a los "blancos, educados y occidentalizados" el rol de dadores y a los "indígenas pobres e ignorantes" el de receptores. Este modelo asistencialista tuvo una recepción ambigua en las comunidades, si por una parte fue percibida como una ayuda importante para la transformación social y cultural de las comunidades; por otra, fue considerada como una estrategia que generaba la dependencia de las comunidades de los conocimientos técnicos y los proyectos de desarrollo (Castellnuovo Biraben, 2010). Pese a las limitaciones, los espacios generados por estas organizaciones constituyeron para las comunidades instancias claves de aprendizaje y de formación, en las que adquirieron habilidades y capacidades nuevas para manejar los discursos estatales y no estatales. Asimismo, las primeras experiencias de los maestros bilingües fueron sustentadas por las ONG y los primeros materiales pedagógicos en educación intercultural. Cuando los pueblos originarios aprendieron a manejar diversas herramientas para la formulación y puesta en marcha de proyectos, comenzaron a demandar una participación más horizontal, y las ONG han debido dar respuesta a estas demandas, generando nuevas estrategias de trabajo.

En cuanto a las universidades públicas ${ }^{14}$ nos centraremos en la Universidad Nacional de Salta (UNSa), la cual tanto en su Sede Central (Salta Capital) como en la Sede Regional Tartagal recibe una creciente matrícula de alumnos pertenecientes a comunidades originarias, particularmente esta última corresponde a la región con la mayor población y diversidad étnica de toda la provincia. En temas de memoria, historia, educación e identidad destacan la labor del Centro de Investigaciones Sociales y Educativas del Norte argentino (CISEN) de ambas sedes, de las carreras de Antropología de la sede central y Ciencias de la Educación (ambas sedes) y recientemente del Instituto de Investigaciones en Ciencias Sociales y Humanidades (ICSOH-CONICET) de la Sede Central. Desde estos espacios se han propuesto diferentes proyectos de extensión, voluntariado e investigación.

Históricamente las relaciones entre la Universidad y las comunidades indígenas en Argentina han transitado entre un paradigma clásico (verticalista, jerárquico, objetivista) y un paradigma del "buen salvaje" (paternalista, relativista, militante), expresado en los discursos institucionales, las lógicas de financiamiento, la formulación y puesta en práctica de los proyectos (Casimiro Córdoba, 2016). Lamentablemente, en ambos casos, la práctica investigativa responde a un modelo de extracción de datos sin mayores devoluciones a la comunidad, mientras que la extensión supone la escasa participación de las comunidades en el diagnóstico social y la formulación de las actividades, puesto que los proyectos suelen llegar a las comunidades con propuestas construidas desde la Universidad. Estas posturas tradicionales dificultan el intercambio entre pares, y son pocos los casos en los que las comunidades se involucran en el proceso de investigación o escritura.

Sin embargo, existe una tercera posibilidad de trabajo que busca romper con la categorización vertical científico-centrista y con el relativismo inocente para avanzar hacia un trabajo en red para la coproducción de conocimientos colectivos y críticos. Este modelo se apoya en la metodología de la Investigación Acción Participativa, IAP, (Fals Borda, 2009; Montero, 2007) ${ }^{15}$ y en la ecología de saberes $\left(\right.$ Santos, 2006) ${ }^{16}$ que habilitan un diálogo más horizontal entre los saberes occidentales y los comunitarios. Bajo esta luz, las relaciones de extensión se entienden como una negociación de saberes y haceres, una co-construcción de conocimientos y estrategias, donde la universidad sugiere y acompaña, pero no decide verticalmente qué es lo mejor o deseable para los/as otros/as.

\section{Tres experiencias de extensión universitaria}

Seleccionamos aquí tres experiencias que ilustran el trabajo extensionista de la UNSa, en las que se busca revertir las relaciones tradicionales de extensión partiendo de necesidades y demandas previamente identificadas y formuladas por la propia comunidad. En estos casos, la Universidad se enfocó en gestionar recursos y estrategias para acompañar los procesos comunitarios.

Una experiencia arquetípica se realizó en 2001 en la Comunidad de Yacuy en articulación con el CISEN (de la UNSa Sede Tartagal y Central) la Iglesia local a través de ENDEPA, el Ministerio de Educación de la provincia y Yacimientos Petrolíferos Fiscales (YPF). En este proyecto participaron docentes universitarios, técnicos del Ministerio Provincial, agentes de la Iglesia, jóvenes, ancianos y maestros bilingües de la comunidad. El trabajo incluyó talleres
13) Entre las vinculadas a la Iglesia Católica, como ENDEPA y la Fundación para el Desarrollo en Justicia y Paz (FUNDAPAZ), y las creadas por la iniciativa de técnicos o profesionales, tales como el Instituto de Desarrollo Social y Promoción Humana (INDES),
Instituto de Cultura Popular (INCUPO) Asociación para el Desarrollo (ADE) y Asociación Regional de Trabajadores en Desarrollo (ARETEDE), entre otras. 14) Cabe mencionar la presencia de la Universidad Nacional de San Martín (UNSAM) y de la Universidad de Buenos Aires (UBA) a través de sus investigadores y algunos proyectos de extensión.

15) La IAP es modelo de investigación que se basa en la acción en tanto apunta a la transformación de la realidad poniendo la ciencia al servicio de las comunidades y en la participación dado que existe un proceso de co-construcción del conocimiento que involucra tanto a los investigadores como a las comunidades, desde el punto de vista epistemológico la IAP supone dos sujetos de conocimiento 
de apoyo escolar por tutorías para los alumnos de la escuela de la comunidad y la capacitación de maestros, auxiliares bilingües y jóvenes bilingües. A través de diferentes actividades tales como entrevista a los ancianos, relevamiento de croquis, leyendas, cuentos, etc. se logró reconstruir la historia comunitaria. Los resultados de este trabajo se vieron plasmados en el libro "Todos juntos busquemos nuestra historia" (2009), publicado en castellano y guaraní con el apoyo de YPF. El libro contribuye a la etnohistoria guaraní y a la revitalización de la lengua nativa, y en ese sentido, marcó un modelo a seguir para otras comunidades e inspiró proyectos posteriores.

La articulación de varias instituciones locales le dio continuidad a esta iniciativa, luego de un segundo proyecto el tejido social conformado continuó formulando actividades educativas y ampliando sus redes hacia actores nacionales e internacionales. Los jóvenes guaraníes que participaron fueron reconocidos por su comunidad y los maestros bilingües se vieron fortalecidos en su formación, algunos de ellos comenzaron a dictar cursos y charlas en lengua y cultura guaraní en espacios universitarios y no universitarios. Yacuy ha logrado un diferencial en términos organizativos respecto de otras comunidades, es así que ha participado de muchos proyectos posteriores como de investigaciones académicas.

Desde 2009-2011 se desarrolló el proyecto "Tendiendo puentes. Escuela, Comunidad y Universidad" financiados por el Programa de Voluntariado Universitario del Ministerio de la Nación y coordinado por docentes y estudiantes de la carrera de Antropología de la UNSa (Sede Central). Del mismo participaron alumnos criollos, guaraníes, chané y wichí del último año de secundaria de la Escuela Familiar Agrícola (EFA) № 8178 (Aguaray, Salta). El proceso de trabajo incluyó una fase de diagnóstico realizado con la comunidad educativa a partir de la cual se registraron las demandas estudiantiles vinculadas a las dificultades de expresar su identidad originaria dentro de la escuela (no hay maestros bilingües en el nivel secundario). A lo largo del proyecto se desarrollaron talleres artísticos y recreativos con los estudiantes tendientes a recrear la memoria e identidad desde la perspectiva juvenil. A través de los autorretratos los alumnos plasmaron en el papel la representación de sí mismos, la identidad étnicacomunitaria se trabajó a través del croquis y la historieta de sus pueblos y la identidad juvenil a través de un boletín escolar.
Debido a las complicaciones presupuestarias y a la distancia geográfica (de 5 a 6 horas de viaje) el proyecto se fue desarrollando de forma intermitente con pocas intervenciones al año que concentraban muchas actividades. Al finalizar este proyecto se formuló otro que buscara continuar la línea de trabajo, pero en una tercera convocatoria se optó por espacios más acordes a los presupuestos. ${ }^{17}$ En este caso, una vez terminado el último año del secundario, los jóvenes iniciaban sus familias, carreras y/o trabajos y no volvían a la escuela; por su parte, los estudiantes universitarios, continuaban sus caminos académicos y laborales. Una vez terminados los proyectos, los tejidos sociales no continuaban retroalimentándose y, sin embargo, esta experiencia marcó y acompañó los procesos de acercamiento a la cultura y la memoria de los estudiantes y una instancia de inflexión en la formación de los estudiantes universitarios.

Una tercera experiencia (en curso) se denomina "Encuentro de saberes entre maestros bilingües guaraníes. Formas de enseñar la lengua y la cultura" financiada desde la Secretaría de Extensión de la UNSa El antecedente inmediato de este proyecto es el "Primer Taller de Lengua y Cultura guaraní, la perspectiva de los maestros bilingües" desarrollado en 2016 a partir de la articulación entre la Universidad Nacional de Salta, la Comunidad Guaraní de Peña Morada y un grupo de maestros bilingües guaraníes autoconvocados. Allí se comenzó a generar un nuevo tejido social entre estudiantes, investigadores, maestros y caciques, en el cual se volcaron las problemáticas y demandas concretas respecto de la EIB. El proyecto de extensión fue una de las estrategias elegidas para generar respuestas colectivas, junto con determinaciones como la conformación de la mencionada Confederación de Docentes Guaraníes (CDG) de los Departamentos de Orán y Tartagal y la escritura de una historia regional. El proyecto de extensión, del que algunos de los maestros son coescritores, se propone acompañar el proceso organizativo de la CDG, aportar a la reactivación y fortalecimiento de la lengua y cultura del pueblo guaraní en la región, y contribuir al desarrollo de la EIB.

La primera actividad del proyecto de extensión es el curso de capacitación en Lengua y Cultura Guaraní destinado a docentes y estudiantes de Salta capital que cuenta con el apoyo del Ministerio de Educación de la provincia, la escuela de Antropología y el ICSOH-CONICET. Está a cargo de dos maestros bilingües guaraníes del departamento San Martín y busca cuestionar la lógica en interacción (Montero, 2007). 16) La ecología de saberes es una propuesta epistemológica que supone que no hay ignorancia ni saber en general, sino que toda ignorancia desconoce cierto saber y todo saber es la superación de una ignorancia en particular. De este modo, todos los saberes (académicos, comunitarios, instituciones, culturales, etc.) se nutren en un reconocimiento muto e intercambio horizontal. El conocimiento se produce entonces en un diálogo y traducción intercultural.
17) Pese a las mejoras en la última década, los programas de voluntariado y de extensión poseen escasos recursos, las condiciones materiales no son un tema menor, muchas veces producen dificultades que desgastan los tejidos sociales. 


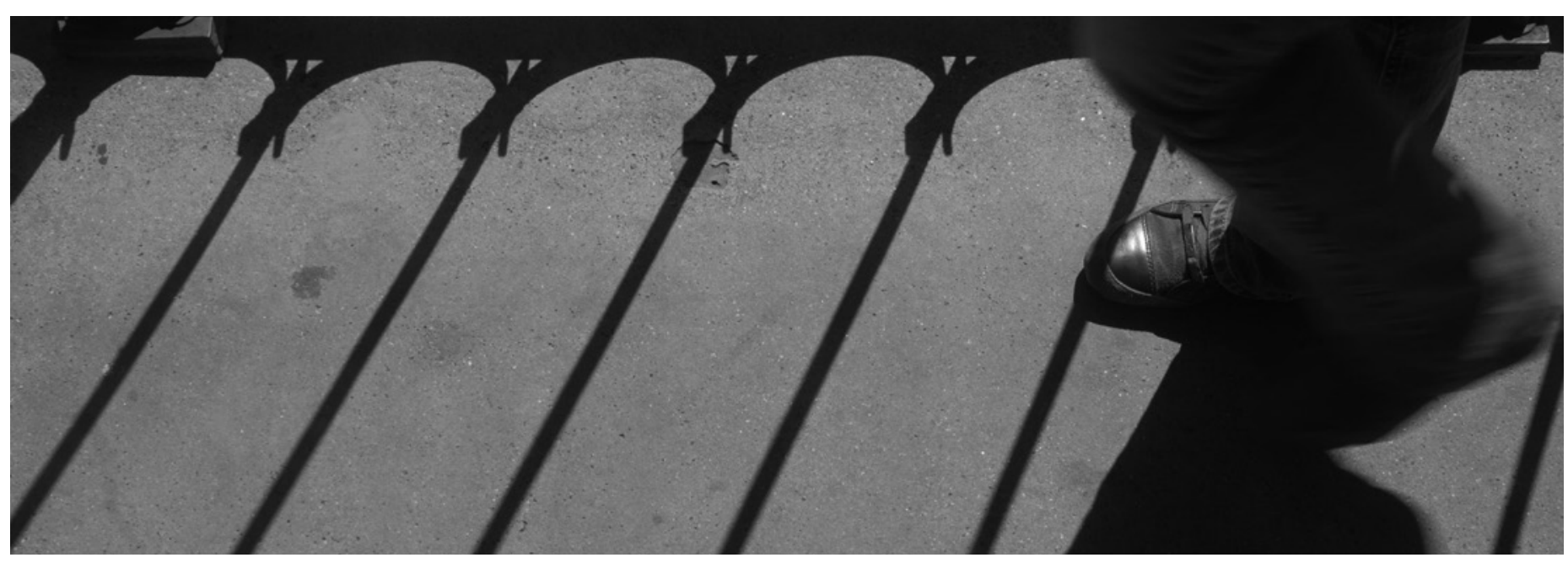

(C) Hugo Pascucci

tradicional de las capacitaciones: ¿quiénes capacitan y quiénes "reciben" las capacitaciones? Que sean los propios maestros bilingües quienes impartan sus saberes lingüísticos y culturales, cambia los lugares de enunciación de estos actores y estimula el dialogo intercultural. El trabajo en red es una de las estrategias para gestionar espacios y recursos (dado el limitado presupuesto del proyecto), y se planifican otros cursos y talleres a ser dictados en las propias comunidades articulando con los caciques,

organizaciones, las ONG, maestros bilingües y jóvenes de la región. Estas experiencias ilustran las condiciones de trabajo en proyectos de corto y largo plazo, sus logros y sus limitaciones, en líneas generales las relaciones de la Universidad con las comunidades son difíciles de sostener, particularmente en los casos en que los investigadores, extensionistas o voluntarios no habitan en la región. En efecto, son los proyectos que articulan con actores locales los que mejores resultados logran, puesto que acompañan tejidos sociales que luego pueden seguir operando. Sin embargo, pese a que la presencia de la universidad pública es limitada en el trabajo territorial, cumple un rol muy importante en la acumulación de experiencias sociales, la activación y fortalecimiento de procesos individuales y colectivos, la articulación de actores y la gestión de respuestas colectivas.

\section{Los maestros bilingües: normatividad y roles sociales}

Un actor social clave tanto en las comunidades y la escuela como en las organizaciones intermedias es el maestro bilingüe, categoría social que desde su emergencia hasta la actualidad ha atravesado procesos de normativización y de resignificación social.

En la provincia de Salta las primeras experiencias de incorporación de traductores indígenas en el aula datan de la década de los ' 80 , sin embargo, recién en 1991 fue oficialmente instituido bajo la figura de Auxiliar del Docente en Lengua Aborigen a través del Decreto Provincial $N^{\circ} 1.488$, que lo entendía como un actor que conecta y traduce dos mundos. En 2008 la Resolución N 460 redefinió el cargo como Docentes Auxiliares en Cultura y Lengua Aborigen, bajo el modelo de pares pedagógicos, es decir, dos docentes en el aula: uno indígena hablante de lengua nativa y uno no indígena hablante de castellano. ${ }^{18}$ Este cambio de nombres no fue inocente, sino que buscaba redefinir el perfil del maestro bilingüe, pasando desde un rol técnico circunscripto a la traducción, hacia un rol docente más involucrado en la enseñanza y el aprendizaje ya no solo de la lengua sino también de la cultura nativa. Este giro discursivo del Estado provincial dio un nuevo marco de acción para el trabajo áulico, sin embargo, no fueron determinadas con claridad las tareas y funciones concretas de los maestros bilingües
18) No obstante, el "par pedagógico" se caracteriza por una relación asimétrica entre el docente no indígena y el indígena. Este último desempe- ña un rol subalterno, "mero auxilio frente al docente criollo portador de la jerarquía y el saber" (Bonillo, Jalil y Quiroga, 2011). 


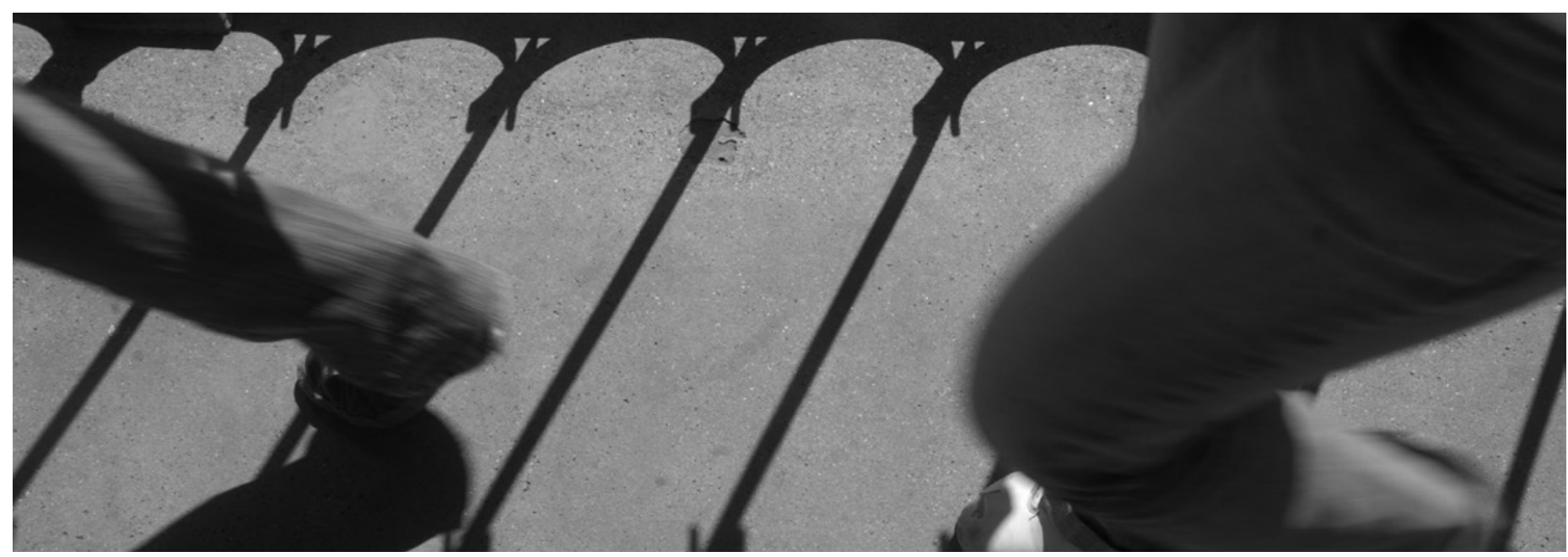

(C) Hugo Pascucci

ni los contenidos específicos que deben desarrollar. Es por ello que aún existe cierta ambigüedad respecto de su rol concreto, siendo las dinámicas sociales locales las que finalmente lo han definido. De aquí que, a pesar de su denominación legal como docente auxiliar, utilizamos en este trabajo la categoría social de maestros bilingües reivindicada por los mismos actores.

A lo largo de tres décadas se pueden identificar algunas instancias generales del proceso de inserción de los maestros bilingües en el sistema educativo. Un momento "fuera de las aulas" en el que éstos no podían trabajar debido a los prejuicios de los docentes no indígenas acerca de su presencia y pertinencia en el aula; y luego una instancia "dentro del aula" que transitó desde un rol de meros traductores de consignas hacia la asistencia en clases y el dictado de una materia propia. Estas fases no se sucedieron de manera acabada en todas partes, puesto que las condiciones concretas de trabajo dependen de la mayor o menor disposición de cada comunidad educativa.

En la actualidad algunos maestros siguen "fuera del aula" realizando funciones de ordenanza y a otros se les asigna tareas no docentes "están para cuidar los chicos cuando la maestra no viene". ${ }^{19}$ En algunas escuelas los maestros bilingües trabajan en el nivel inicial y básico (hasta $3^{\circ}$ grado) en otras lo hacen hasta el $7^{\circ}$ grado, pero en ningún caso se desempeñan en el nivel medio. Los permisos para capacitaciones, las posibilidades de inferir en los contenidos dictados, la participación en las carteleras, los actos y demás actividades cotidianas de la escuela, varían de acuerdo a las relaciones entre los docentes indígenas y no indígenas. Permeadas por la discriminación, la estigmatización y las luchas por el prestigio, estas relaciones suelen tener instancias de conflicto. No obstante, hay algunos casos de articulación y solidaridad entre los docentes e incluso la producción conjunta de materiales pedagógicos. Este es el caso de las tres comunidades aquí estudiadas, en las que los equipos de maestros no solo han podido desarrollar experiencias de enseñanza y aprendizaje de la lengua y cultura guaraní, sino que además han podido producir materiales pedagógicos. A su vez, fuera de la escuela estos maestros cumplen funciones muy diversas vinculadas a la vida comunitaria y la cultura. De esta forma, el rol que los maestros bilingües cumplen en las comunidades y escuelas se va dibujando a partir del entramado social cotidiano. En líneas generales, hemos detectado algunos campos de intervención fuera del aula:

a) El maestro bilingüe como líder comunitario, puesto que es canalizador de las demandas colectivas a través de la gestión institucional, entablando comunicación entre las comunidades

19) Comentario registrado en el marco del "Primer Taller de Lengua y Cultura guaraní, la perspectiva de los maestros bilingües", Peña Morada, septiembre de 2016. 
y actores, políticas y discursos estatales. En este sentido, se orientan las participaciones activas de los maestros en las organizaciones intermedias vinculadas a la educación tales como el CEAPI o la CDG, algunos maestros fueron reconocidos como mburivichas (líderes) en sus comunidades y otros llegaron a ocupar el rol de caciques (autoridad o jefe comunitario).

b) El maestro como promotor de la lengua y cultura guaraní, porque su participación en actividades de gestión, promoción y valorización de la lengua y la cultura ha sido patente en espacios tales como los centros comunitarios, los medios de comunicación local, las fundaciones y las universidades.

c) El maestro como escritor, productor de material pedagógico tanto destinado a las aulas como de difusión general, productores de discursos acerca de "lo guaraní", escritores de la historia comunitaria, narradores de los memoria colectiva e intelectuales de la lengua que se orientan a fijar las formas de escribir, los modos de decir y los contenidos para la enseñanza.

De esta manera, el maestro bilingüe se encuentra en una posición privilegiada para la transformación educativa de su pueblo, pudiendo articular con actores de diversos espacios, tales como la Universidad, las ONG y el Estado.

\section{Tres maestros bilingües de Salta}

Presentamos aquí las experiencias educativas de tres maestros bilingües (a los que llamaremos Adrian, René y Gabriel) que actualmente ocupan lugares estratégicos dentro de la enseñanza de la lengua nativa en las mencionadas comunidades de Yacuy (Tartagal), Tuyunti (Aguaray) y Cherenta (Tartagal). Buscamos aquí trazar los caminos individuales y colectivos y mostrar cómo estos intelectuales originarios, gestores y promotores de la EIB, interactuaron con diversos actores, particularmente con la universidad pública.

\section{*Adriana}

Adriana es de la comunidad chané Tuyunti (Aguaray, Salta), su madre es guaraní y sus abuelos eran aymaras. El grupo familiar migró hacia la Argentina en busca de trabajo y se quedó a vivir en Tuyunti. Allí su joven madre conoció a un hombre mapuche y tuvieron a Adriana. Para ella su historia familiar da cuenta de la interculturalidad de nuestro país y de cómo las historias de los pueblos están conectadas. $^{20}$
La educación formal en su comunidad inició con los franciscanos, quienes llegaron en 1930 y fueron los primeros en alfabetizar a la población de la zona. En 1947 se abrió la primera escuela del pueblo; cuando Adriana ingresó a la misma tenía ya 6 años, su lengua materna era el guaraní y fue allí donde aprendió el chané, pues todos sus compañeros de la escuela lo hablaban. En esa época primaba un modelo castellanizante y la repitencia entre los niños de pueblos originarios era muy común. Esto preocupó a la Hermana Micaela, quien convocó a ENDEPA para iniciar el camino de la EIB en Tuyunti.

Adriana inicia su trayectoria en EIB como traductora cuando todavía iba al colegio secundario, al egresar comienza a trabajar como maestra bilingüe en la escuela de Tuyunti San Miguel Arcángel No 4736 (Aguaray, Salta). Por entonces la lengua chané no tenía una escritura, por lo que fue necesario elaborarla a partir de un trabajo con los ancianos de la comunidad, contaron para ello con el apoyo de docentes y expertos llegados de Bolivia y Paraguay. Adriana participa de estos procesos y gracias al apoyo de ENDEPA comienza a realizar cursos de capacitación y formación en EIB que la llevan a intercambiar y aprender del pueblo Qom del Chaco. Esas experiencias son volcadas en el trabajo en su comunidad. Para 1994 el equipo de maestros bilingües del que Adriana forma parte edita un libro para el primer ciclo de la primaria "Jekue jaiko jev. Volver a vivir", escrito en castellano y chané este libro compila cuentos y leyendas: "la idea era escribir sobre aquellos elementos de la cultura chané que pueden ser alfabetizados, por eso seleccionamos los aspectos más folclóricos que podían ser atractivos para los mas pequeñitos". ${ }^{21}$ Adriana continúa su formación en la UNSa (Sede Tartagal), allí estudia la licenciatura en Ciencias de la Educación donde accede a herramientas conceptuales y metodológicas que le permiten complejizar su mirada acerca del contexto educativo chané. Sus preocupaciones la llevan a relacionarse con el CISEN, del cual es miembro actualmente, y entra en contacto con proyectos de investigación y extensión y la bibliografía académica sobre su pueblo y sobre la EIB.

En el año 2013 Adriana presenta su tesis de grado "Propuestas didácticas en la práctica pedagógica de EIB frente a la ausencia de saberes ancestrales en el espacio curricular de Ciencias Sociales", la defiende en castellano y en chané. Al poco tiempo es elegida Directora de la escuela de su comunidad donde comienza a aplicar diferentes estrategias pedagógicas para fortalecer la lengua y la 
cultura chané en los niños, tales como cursos de cocina tradicional, artesanía y folclore chané. Los alumnos tienen cartillas de medicina ancestral como una forma de afianzar su identidad y los adolescentes chanés tienen una atención especial, porque por años sufrieron discriminación a tal punto que dejaron de hablar su lengua materna. Así en la escuela se refuerza "lo chané" como identidad cultural diferenciada, no obstante también se realizan actividades interescolares durante la semana del aborigen tendientes a tender puentes con el mundo guaraní del cual son parte.

A lo largo de su trayectoria Adriana se convirtió en un referente comunitario, en el año 2007 recibió el premio "Maestro llustre 2007" otorgado a nivel nacional en reconocimiento por sus aportes a la EIB. Asimismo, es integrante del CEAPI como representante del pueblo chané. Junto con otros maestros bilingües ha participado de la producción de materiales pedagógicos locales, en coautoría con investigadores ha escrito artículos científicos y con el apoyo del Ministerio de Educación de la Nación ha publicado materiales de difusión. Su comunidad sigue siendo foco de proyectos e intervenciones de diferentes actores, entre los que destaca el proyecto "Comunidades de aprendizaje" llevado a cabo en colaboración entre investigadores del CISEN (Sede Tartagal), técnicos del Ministerio de Educación de la Provincia, el Centro de Implementación de Políticas Públicas para la Equidad y el Crecimiento (CIPPEC) y la empresa brasilera Natura.

\section{* René}

René es de la comunidad Tupi guaraní San José de Yacuy en el área rural de Tartagal, la misma se conformó por refugiados de la Guerra del Chaco provenientes del Isoso boliviano, región en la cual aún conservan parientes con quienes mantienen fluidas relaciones. La comunidad de Yacuy se encuentra en un entorno rural, mantiene muchas prácticas del ñande reko ${ }^{22}$ tradicional guaraní y realizan el arete guasú, ${ }^{23}$ la fiesta más importante del pueblo guaraní que muchas comunidades vecinas ya han dejado de celebrar. La lengua es sumamente vital y allí René aprendió el guaraní isoseño o tupi guaraní como lengua materna.

En la década de los ' 50 se instaló la escuela de la comunidad y en ella los niños aprendían a hablar y escribir en castellano. Hacia los años '80 el modelo educativo hegemónico era muy cuestionado desde varios sectores y, como mencionamos anteriormente, fue ENDEPA quien inició las primeras experiencias hacia una educación intercultural en esta región. En los ‘90 se logró el nombramiento del primer auxiliar bilingüe de Yacuy y en los años 1991 y 1993 se realizó allí una serie de capacitaciones para docentes y auxiliares bilingües organizadas por ENDEPA. Entidad que también organizó en 1994 un encuentro clave en la comunidad de San José de Yacuy que convocó a otras comunidades ava guaraní y chané de la región y contó con la presencia del jesuita Bartolomeu Melia, reconocido antropólogo y lingüista del Paraguay. Asimismo, Yacuy fue el lugar elegido para encuentros con especialistas en EIB del Chaco en 1997 y de un curso de capacitaciones en guaraní con técnicos de EIB en Bolivia e investigadores del Consejo de Investigación de la unas. René, junto a los primeros maestros bilingües, se formó entonces en un contexto sumamente activo en el que los intercambios trascendían las fronteras provinciales y nacionales.

Como hemos señalado, a partir de 2001 se ejecutó en Yacuy un proyecto paradigmático en el que se desarrollaron capacitaciones a docentes, auxiliares y jóvenes guaraníes y se logró la publicación de un libro en castellano y guaraní. René fue el responsable de enseñar el guaraní a los técnicos del Ministerio e investigadores que participaron. Gracias a su experiencia y formación comenzó a integrar el equipo de investigación y actualmente es miembro del CISEN Sede Tartagal. Asimismo, ha realizado estudios terciarios y diversas capacitaciones en EIB.

Su experiencia docente abarca no solo el nivel primario y secundario sino también el superior, pues ha dictado cursos de Lengua y Cultura guaraní en un Terciario y en la UNSa (Sede Tartagal). René es un gran promotor de la lengua guaraní y ha impartido cursos y charlas fuera del sistema educativo, en sus cursos emplea materiales provenientes de Bolivia y Paraguay, junto con apuntes propios, pues sostiene que "todos somos hermanos y a pesar de las diferencias cuando hablamos nos entendemos, entonces a mí me gusta hablar de una sola gran familia guaraní”. ${ }^{24}$ Actualmente, Yacuy sigue participando de diferentes proyectos y vinculando diversas instituciones; es una de las tres comunidades elegidas para el desarrollo del modelo educativo alternativo español "Comunidades de aprendizaje" junto con Tuyunti y Pichanal. La comunidad es gestora de numerosas iniciativas y algunas son extendidas a otras comunidades de la zona con el objeto de afianzar los lazos del pueblo guaraní.

La familia de René siempre ha estado vinculada al liderazgo comunitario, su abuelo fue el cacique fundador de Yacuy y sus hermanos son líderes reconocidos, y esto ha marcado su trayectoria
22) El termino ñande reko se puede traducir como "nuestro modo de ser" o "nuestro modo de vida". 23) El arete guasiu o fiesta grande en el tiempo verdadera es una celebración de los pueblos guaraníes en la que se agradece la cosecha del maíz y el fin del año agrícola, en ella cobran importancia los antepasados de la comunidad, la música y la danza grupal. 24) Entrevista a René, Tartagal, abril de 2016. 
personal como referente guaraní en el área de educación. En efecto, René ha formado parte del CEAPI desde su fundación y actualmente participa activamente en la conformación de un CEAPI Provincial: "Ya es tiempo de una organización provincial, donde podamos tener más representantes indígenas por pueblo y que puedan articular con sus pares del CEAPI nacional". ${ }^{25}$ Desde este lugar ha convocado a Adriana y a otros maestros bilingües para traducir canciones, leyendas, cuentos, recetas, etc., publicados por el Ministerio de Educación de la Nación en la serie "Con nuestra voz cantamos: Escritos plurilingües de docentes, alumnos, miembros de pueblos originarios y hablantes de lenguas indígenas" (2015). En coautoría con otros investigadores, René ha publicado artículos tanto sobre la lengua y cultura guaraní como también acerca de la EIB.

\section{* Gabriel}

Gabriel "Tito" Domínguez es de la comunidad ava guaraní Misión Cherenta (Tartagal, Salta), allí aprendió guaraní y castellano. Realizó la primaria en su comunidad, en esa época no contaban con un maestro auxiliar y las clases se dictaban en castellano. Luego viajó a la vecina provincia de Jujuy para completar los estudios secundarios y fue allí donde entró en contacto con las comunidades guaraníes de la zona en pleno proceso organizativo. Esa experiencia lo llevó a capacitarse en Educación Bilingüe en Camiri, Bolivia, donde se formó con el modelo educativo, los materiales pedagógicos y las organizaciones bolivianas. Al regresar a la Argentina realizó sus primeras experiencias docentes en Yacuy (Tartagal) para luego retornar y comenzar a trabajar como maestro bilingüe en la escuela de su comunidad.

En el marco del Programa Nacional de Educación Intercultural Bilingüe (PNEIB), Tito participó junto a otros maestros bilingües y docentes de la elaboración del primer material pedagógico de la escuela de Cherenta. Es así que, en el año 2008, luego de muchos esfuerzos, se imprimió un libro: Tëtaguasu guaraní itekoasa vae, Historia de un pueblo guaraní, y un diccionario: Ñande miari icavi vaëra, Para expresarnos mejor en guaraní. Durante el año 2012, con el apoyo económico del Ministerio de Educación, Ciencia y Tecnología, se produjo un nuevo material destinado al trabajo áulico, Ayemboe amongeta jare aikuatia ñande ñee pe, Aprendiendo a leer y escribir en guaraní. En el año 2015, el mismo equipo de maestros bilingües emprendió la grabación de un CD con historias y relatos de los ancianos de la comunidad y el Himno Nacional cantado en ava guaraní por los niños de la escuela.

Por otra parte, Gabriel ha participado, junto a otros maestros bilingües, en la traducción de algunos cuentos y leyendas publicados por el Ministerio de Educación y ha contribuido con investigaciones científicas en la zona. Sin embargo, su contacto con la Universidad ha sido escaso debido a que investigadores y extensionistas suelen llegar a Cherenta para realizar breves entrevistas y desarrollan su trabajo más fuerte en las comunidades rurales. Aunque en 2016 Gabriel comenzó a interactuar con investigadoras de la UNSa (Sede Central) en talleres, entrevistas e intercambios que desembocaron en la formulación del mencionado proyecto de extensión "Encuentro de saberes..." del cual es coescritor y miembro activo. Desde estos espacios, Gabriel, junto a su colega Guillermo, dicta un curso de capacitación en "Lengua y cultura guaraní" en la ciudad de Salta y proyecta cursos similares en el interior. Desde ese curso, estos maestros abogan por una escritura unificada del guaraní sin desconocer las variantes regionales.

Este acercamiento con la Universidad se da en paralelo al proceso organizativo de los maestros bilingües guaraníes de la Ruta 34, que tiene como puntos clave un encuentro desarrollado a mediados de 2016 con el apoyo del Instituto de Pueblos Indígenas de Salta (IPIS) y el mencionado "Taller de EIB la perspectiva de los maestros" en articulación con la Universidad. Todos estos espacios contribuyeron para estimular un nuevo tejido social. Para febrero de 2017, los maestros autoconvocados conformaron la Confederación de Docentes Guaraníes CDG (Oran y Tartagal), Tito fue elegido su primer presidente y desde ese momento inició una nueva etapa en su trayectoria, comenzó a caminar por las comunidades charlando con los caciques, visitando las escuelas y reuniendo a los maestros bilingües. Con el acompañamiento de las investigadoras de la UNSa empezó a visitar instituciones tales como la coordinación de EIB de la provincia de Salta o el ICSOH-CONICET. Sus expectativas están enfocadas en la recopilación de los materiales pedagógicos y las experiencias en función del armado de un currículo guaraní. ${ }^{26}$ Estos maestros bilingües han articulado con la Universidad desde diferentes lugares, ya en el ámbito de la formación (alumno o docente) como en el de la investigación (en tanto objetos y sujetos de conocimiento) y en el de la extensión (como destinatarios e integrantes de los proyectos). La presencia y agencia de la Universidad en cada caso es diferente; sin embargo, observamos un proceso de subjetivación caracterizado por la adquisición de nuevos saberes y capitales culturales, la incorporación de un lenguaje específico y de la lógica y códigos del ámbito intelectual. En ese sentido, la Universidad ha tendido a reproducir sus esquemas al incorporar a nuevos sujetos dentro de sus lógicas. Si bien estos maestros bilingües se empoderaron, lo clave ha sido el

25) Ídem.

26) Entrevista a Gabriel, Salta capital,

marzo de 2017. 
Las relaciones entre la Universidad y las comunidades indígenas en Argentina han oscilado desde un modelo clásico-objetivista a uno relativista-paternalista, atravesado por estructuras de clasificación social y racial

proceso de socialización de los aprendizajes, es decir, la capacidad de volcar sus conocimientos y experiencias a la comunidad. Como hemos visto, la comunidad universitaria contribuyó a la formación de estos líderes e intelectuales indígenas con quienes estableció lazos duraderos, pero esto generó dos tipos de problemas: a) una concentración de proyectos en las mismas comunidades y b) una relativa desigualdad entre los actores que logran insertarse en la lógica universitaria y aquellos que quedan por fuera de esta red. En ese sentido, podemos cuestionarnos: ¿la Universidad es capaz de gestar dispositivos que generen un impacto comunitario e intercomunitario sin reproducir desigualdades?

\section{¿EI cierre? La Universidad para pensar las estrategias colectivas} La propia noción de extensión ha sido ya muy debatida en Latinoamérica, especialmente en los años ‘60 y, sin embargo, continúa siendo utilizada dada la fuerza política que posee particularmente en relación con la investigación más academicista. Desde el retorno a la democracia (1983), este ha sido un campo de intervención asociado al compromiso social de la universidad pública con la comunidad. En el último período (2003-2015) destacan las políticas sociales del kirchnerismo ${ }^{27}$ y se activaron procesos de resemantización tanto de la universidad pública y la extensión como de la ciencia pública. Desde un lenguaje político propio fueron emergiendo discursos asociados a la "inclusión social", la "ampliación de derechos" o la "democratización de la educación". Las relaciones entre la Universidad y las comunidades indígenas en Argentina han oscilado desde un modelo clásico-objetivista a uno relativista-paternalista, atravesado por estructuras de clasificación social y racial. En este trabajo sostenemos que la extensión puede ser resignificada en un tercer modelo de trabajo basado en la Investigación Acción Participativa (Fals Borda, 2009; Montero, 2007) y en la ecología de saberes (De Sousa Santos, 2006) que habilitan un intercambio de saberes y haceres en la co-construcción de conocimientos y estrategias colectivas. Las experiencias reseñadas van en esa línea de trabajo hacia una universidad pública participativa y acompañante de procesos que ya están dados en el territorio.

Las trayectorias educativas de los tres maestros bilingües nos muestran que la Universidad es un actor más en el terreno que aporta tanto a la formación de recursos humanos y líderes, como al acompañamiento de procesos colectivos en marcha. Comprender el entramado de actores e instituciones de los contextos locales, permite posicionar a la universidad pública y analizar el campo de sus intervenciones. Esto ha permitido identificar algunos procesos globales tales como la reproducción de la lógica académica, que incorpora a los intelectuales originarios dentro de la misma. Si postulamos que la relación Universidad-comunidad debe pensarse en los términos de intercambio y negociación de saberes, cabe indagar acerca de los mecanismos que se pueden generar desde la Universidad para incorporar y socializar los saberes culturales originarios.

Otro punto de indagación tiene que ver con los procesos de concentración de las investigaciones y proyectos de extensión en algunas comunidades. Ciertamente esto ha contribuido al
27) Movimiento político que emerge luego de la crisis económica de 2001 cuyos líderes principales fueron los expresidentes Néstor Kirchner (2003-2007) y Cristina Fernández de Kirchner (2007-2010 y 2011-2015). 
empoderamiento de los pueblos originarios, pero al mismo tiempo ha generado diferenciales de poder, en términos organizativos, de algunas comunidades respecto de otras. Al momento de ingresar al terreno cabe a los investigadores indagar estos aspectos, siendo consciente de que sus intervenciones tienen consecuencias sociales, y en muchos sentidos pueden estar reproduciendo desigualdades intercomunitarias.

Asimismo, las trayectorias excepcionales de los maestros bilingües que hemos expuesto aquí los sitúa actualmente en lugares estratégicos para el desarrollo de la EIB, lugares desde los cuales disputan los sentidos sociales de lo guaraní y lo chané frente a la sociedad mayoritaria. Los capitales sociales y culturales que estos actores manejan les permiten interactuar con discursos estatales y no estatales e intercambiar fácilmente con investigadores, a la manera de informantes clave. La transferencia de esos saberes hacia la comunidad no ha sido acompañada por la Universidad, proceso central que permitiría inyectar a la comunidad saberes, habilidades e información sumamente útiles. Se han registrado casos de distanciamiento de estos actores respecto de sus comunidades, en efecto, la CDG ha surgido justamente en tensión con el CEAPI nacional, cuestionando los procesos de circulación de saberes y los mecanismos de participación indígena en los asuntos educativos. Nuestra propuesta es contribuir a la disminución de las brechas de desigualdad entre los diferentes actores, fortaleciendo los esfuerzos colectivos y el trabajo en red. Desde este lugar, podemos pensar la extensión universitaria como espacio de intercambio y producción de conocimientos sociales tendiente a generar horizontalidad inter e intracomunitaria. El desafío es sistematizar las experiencias acumuladas en el área de extensión e investigación, orientando el trabajo hacia el diseño de políticas estatales que emerjan de un trabajo de base comunitaria. Se trata de co-construir respuestas colectivas a las problemáticas y demandas que la comunidad identifica como conflictivas, importantes o críticas. De esta manera, el objetivo es generar un impacto más global en el tejido social como tal y no enfocado en actores o comunidades en particular. El sentido estratégico de ello es sumar los esfuerzos colectivos, articulando los saberes y retroalimentado una red de actores que se impulsen los unos a los otros.

El rol de los extensionistas puede ser justamente propiciar estos espacios de ensayo y co-construcción de respuestas sociales, tendiendo puentes entre las distintas instituciones del espacio social (Estado, escuela, comunidades, etc.). En el caso específico que hemos tratado, resulta inminente aportar a la reactivación y fortalecimiento de la lengua y cultura del pueblo guaraní en la región, y contribuir al desarrollo de la EIB desde un paradigma popular, que apueste a la participación indígena en la toma de decisiones y en la producción de contenidos y materiales pedagógicos, considerando los conocimientos y saberes ancestrales. El objetivo es transformar los lugares de enunciación que actualmente tienen los pueblos originarios frente al Estado nacional y provincial, contribuyendo al empoderamiento de múltiples actores y sectores.

Acompañar los procesos organizativos implica necesariamente un trabajo en red con actores locales, en este sentido, la Universidad puede facilitar estos contactos y velar por intercambios más horizontales. Ello permitiría no solo visibilizar las problemáticas locales en la implementación de la EIB, sino también ampliar las condiciones de posibilidad de estas demandas. La articulación de las organizaciones intermedias indígenas, permitiría sumar experiencias y saberes intercomunitarios, estimular espacios de reflexión intercultural, ampliar la autocrítica del camino andado y generar nuevas propuestas de trabajo que puedan ser aplicadas a todas las comunidades. Acompañar y activar los tejidos sociales en el terreno permitiría, en última instancia, estimular un proceso espiralado ascendente orientado a la revalorización de la lengua y cultura guaraníes. 


\section{6}

El desafío es sistematizar las experiencias acumuladas en el área de extensión e investigación, orientando el trabajo hacia el diseño de políticas estatales que emerjan de un trabajo de base comunitaria

\section{Referencias bibliográficas}

EMGC, Equipo Mapa Guarani Continental (2016). Cuaderno Mapa Guarani Continental: Pueblos Guaranies en Argentina, Bolivia, Brasil y Paraguay. Campo Grande, Brasil: MS. Gráfica Mundial.

Bonillo, M. B.; Jalil M. L. y Quiroga A. del P. (2011). Atravesando el bosque: tramas escondidas de la formación docente regional. En Bazán, M. D. (Comp.), Exploraciones de frontera. (Esbozos pedagógicos interculturales) (pp. 45-56). Salta: Hanne.

Casimiro Córdoba, A. V. (2016). Extensión Universitaria, Comunidades Indígenas y Estado. Trabajo final de la Diplomatura no publicado. CLACSO, Buenos Aires. Casimiro Córdoba, A. V. y Flores M. E. (2016). La Lengua Guaraní en el umbral al Chaco salteño. Diagnóstico y propuestas. Tramas/Maepova, 5(1), 19-38.

Castelnuovo Biraben, N. (2010). Tensiones, contradicciones y disputas: guaraníes y ONG's de desarrollo en el noroeste argentino. Avá, (18), 43-59.

De Sousa Santos, B. (2006). Uma sociologia das ausências e uma sociologia das emergências. En De Sousa Santos, B., A Gramática do Tempo. Para uma nova cultura política (PP. 87-125). Porto: Afrontamento.

Fals Borda, O. (2009). Cómo investigar la realidad para transformarla. En Moncayo, V. M. (Org.), Una sociología sentipensante para América Latina. Orlando Fals Borda (pp. 253-301). Bogotá: Siglo del Hombre Editores y CLACSO.

Hisch, S. (2004). Ser guaraní en el noroeste argentino: variantes de la construcción identitaria. Revista de Indias, LXIV(230), 67-80.

Montero, M. (2007). Hacer para transformar. El método en psicología comunitaria. Buenos Aires: Paidós Tramas Sociales.

Machaca, R. (2011). El camino de la participación indígena en la gestión de la Modalidad de Educación Intercultural Bilingüe. En Serrudo, A. (Coord.). La modalidad de educación intercultural bilingüe en el sistema educativo argentino (pp. 11-16). Buenos Aires: Ministerio de Educación de la Nación Argentina.

Serrudo, A. (2011). De Programa a Modalidad: reflexiones desde la política educativa y los desafíos de la gestión con participación. En Serrudo, A. (Coord.). La modalidad de educación intercultural bilingüe en el sistema educativo argentino (pp. 11-16). Buenos Aires: Ministerio de Educación de la Nación Argentina. 